Volume 3, Issue 2, pages 257-264

\title{
Writer Identification Based on Hand Writing using Artificial Neural Network
}

\author{
Rosalia Arum Kumalasanti ${ }^{1, *}$ \\ ${ }^{1}$ Department of Informatics, Faculty of Science and Technology, \\ Sanata Dharma University, Yogyakarta, Indonesia \\ *Corresponding Author: rosaliasanti@usd.ac.id
}

(Received 26-11-2021; Revised 30-12-2021; Accepted 31-12-2021)

\begin{abstract}
Humans are social beings who depend on social interaction. Social interaction that is often used is communication. Communication is one of the bridges to connect social relations between humans. Communication can be delivered in two ways, namely verbal or nonverbal. Handwriting is an example of nonverbal communication using paper and writing utensils. Each individual's writing has its own uniqueness so that handwriting often becomes the character or characteristic of the author. The handwriting pattern usually becomes a character for the writer so that people who recognize the writing will easily guess the ownership of the related handwriting. However, handwriting is often used by irresponsible people in the form of handwriting falsification. The acts of writing falcification often occur in the workplace or even in the field of education. This is one of the driving factors for creating a reliable system in tracking someone's handwriting based on their ownership.

In this study, we will discuss the identification of a person's handwriting based on their ownership. The output of this research is in the form of ID from the author and accuracy in the form of percentage of system reliability in identifying. The results of this study are expected to have a good impact
\end{abstract}




\section{International Journal of Applied Sciences and Smart Technologies}

Volume 3, Issue 2, pages 257-264

p-ISSN 2655-8564, e-ISSN 2685-9432

on all parties, in order to minimize plagiarism. Identification of handwriting to be built consists of two main processes, namely the training phase and the testing phase. At the training stage, the handwritten image is subjected to several processes, namely threshold, wavelet conversion, and then will be trained using the Backpropagation Artificial Neural Network. In the testing phase, the process is the same as in the training phase, but at the end of the process, a comparison will be made between the image data that has been stored during training with a comparison image.

Backpropagation ANN can work optimally if it is trained using input data that has determined the size, learning rate, parameters, and the number of nodes on the network. It is expected that the offered method can work optimally so that it produces an accurate percentage in order to minimize handwriting falcification.

Keywords: Handwriting, Artificial Neural Networks, backpropagation

\section{Introduction}

The use of communication in the digital era is growing and becomes an important need in various aspects of life. Communication is the essence of humans as social beings in interacting with each other. In general, communication is defined as the process of delivering and receiving messages between two or more people directly or indirectly. In short, it can be concluded that the purpose of communication is to create understanding between two or more parties. One example of how to communicate nonverbally is by writing. Handwriting is still often used for various purposes, such as writing documents or forms, writing letters, and also for signatures. Everyone's handwriting has different characteristics and patterns so that handwriting is considered a unique attribute that is owned by each person or also known as a biometric attribute. The uniqueness of handwriting allows people to be able to distinguish one writing from another according to the characteristics of the pattern.

Handwriting is often considered a trivial thing so many people think that the validity of handwriting is not too important. This causes an increasing number of plagiarism that 


\section{International Journal of Applied Sciences and Smart Technologies}

Volume 3, Issue 2, pages 257-264

p-ISSN 2655-8564, e-ISSN 2685-9432

occurs, especially in the work environment and even in the educational environment. Visually or with the naked eye, humans cannot directly distinguish and identify the owner of the handwriting. Even if humans know that there are differences in the handwriting, they have difficulty in identifying the owner of the handwriting. This is an example of a person's lack of concern for handwriting ownership.

This study aims to protect handwriting as a biometric attribute that is protected by ownership. The handwriting identification system will be built using Backpropagation ANN and supporting parameters. A reliable system is expected to be a solution in minimizing handwriting forgery and providing awareness to all people about the importance of handwritten characters. ANN works like the human brain in identifying an object by studying patterns and storing characteristics for comparison with other objects. The more characteristics that are stored for study, the smarter the ANN will be in identifying.

\section{Research Methodology}

Writing is one of the ways humans communicate with the aim of conveying information using written media. Although the digital era has developed, handwriting is still often used for formal and non-formal activities. Handwriting has become one of the biometric attributes that offers several methods that can be developed in research. Most handwriting identification uses ANN and several other supporting algorithms ANN is used to perform initial analysis and categorize handwritten input images [1]. Each individual's writing has its own characteristics that can make a difference.

2.1. Biometrics and Character of a Person. Biometrics is a science and innovation to describe information from the human body naturally [2]. Biometrics refers to individual differences based on physiological or social attributes. The biometric value itself can be an individual's physiological or social attributes of completeness. A person's handwriting has its own characteristics related to a person's character. Graphology is the science of analyzing handwriting and the characteristics of a person's personality through the extraction of features based on shape [3]. The identification of the four 


\section{International Journal of Applied Sciences and Smart Technologies}

Volume 3, Issue 2, pages 257-264

p-ISSN 2655-8564, e-ISSN 2685-9432

dominant forms of handwriting is divided into several attributes, as shown in table 1 below.

2.2. JST Backpropagation. Artificial Neural Networks (ANN) refers to the paradigm of information processing or computing systems inspired by biological neural networks in the human brain. The system is not biologically identical to the nervous system, but is designed to process information in the same way that the human brain processes information. The network consists of many neurons that are interconnected and work simultaneously to achieve certain goals. Just like the human brain, ANN learns from the object examples presented, so ANN can be configured for an application. The application in question is like data classification or character recognition through the learning process. Figure 1 shows the architecture of ANN

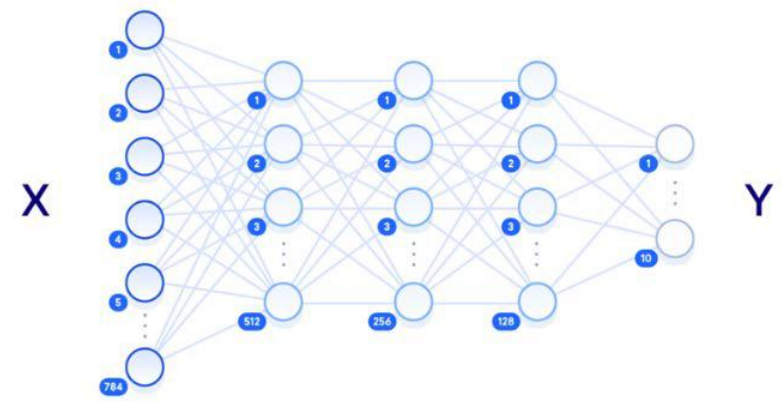

Figure 1. Artificial Neural Network Architecture [4]

Backpropagation is one of the ANN models that has the ability to get a balance between the network's ability to recognize the patterns used during training and the ability to provide the correct response to input patterns that are similar to the patterns used during training. Backporpagation algorithm is an algorithm that can find the minimum error or error in the weights by using gradient descent. The combination of weights that can minimize errors is considered capable of providing solutions to the learning process. Backpropagation has several neurons at each network layer.

Wavelet Transform Preprocessing in this study utilizes wavelet switching to represent the time and shape frequency signal. Wavelet transform is the basis of mathematical tools on several transfer layer functions and produces coefficients that 


\section{International Journal of Applied Sciences and Smart Technologies}

Volume 3, Issue 2, pages 257-264

p-ISSN 2655-8564, e-ISSN 2685-9432

represent signal characteristics [5]. Wavelets provide accuracy and analysis of signals of more than one resolution, which is known as multi-resolution capability. The advantage of this multi-resolution analysis is that features that may not be detected at one resolution can be detected using another resolution [6]. Discrete Wavelet Transform is the choice as an efficient way to be used on images in the form of discrete data. Wavelet has a decomposition level that is useful for obtaining optimal information on an image. Figure 2 below is a level 2 decomposition image.

\begin{tabular}{|c|c|c|}
\hline LL2 & HL2 & \multirow{2}{*}{ HL1 } \\
LH2 & HH2 & \\
\hline LH1 & HH1 \\
\hline
\end{tabular}

Figure 2. Decomposition level 2 [7]

\section{Results and Discussion}

Identification of handwritten images consists of two phases, namely training and testing. A number of samples in the form of handwritten images are used as input data. The greater the amount of input data, the better for ANN in studying patterns, but it is also necessary to consider the amount of time needed for ANN to study it. This identification aims to build an effective and efficient system for users to obtain the identity of the author. Image samples with a certain size also need to be uniform so that the system can easily get the characters in each image with optimal size and results. Figure 3 below is a handwriting identification flow using Backpropagation ANN. 


\section{International Journal of Applied Sciences and Smart Technologies}

Volume 3, Issue 2, pages 257-264

p-ISSN 2655-8564, e-ISSN 2685-9432

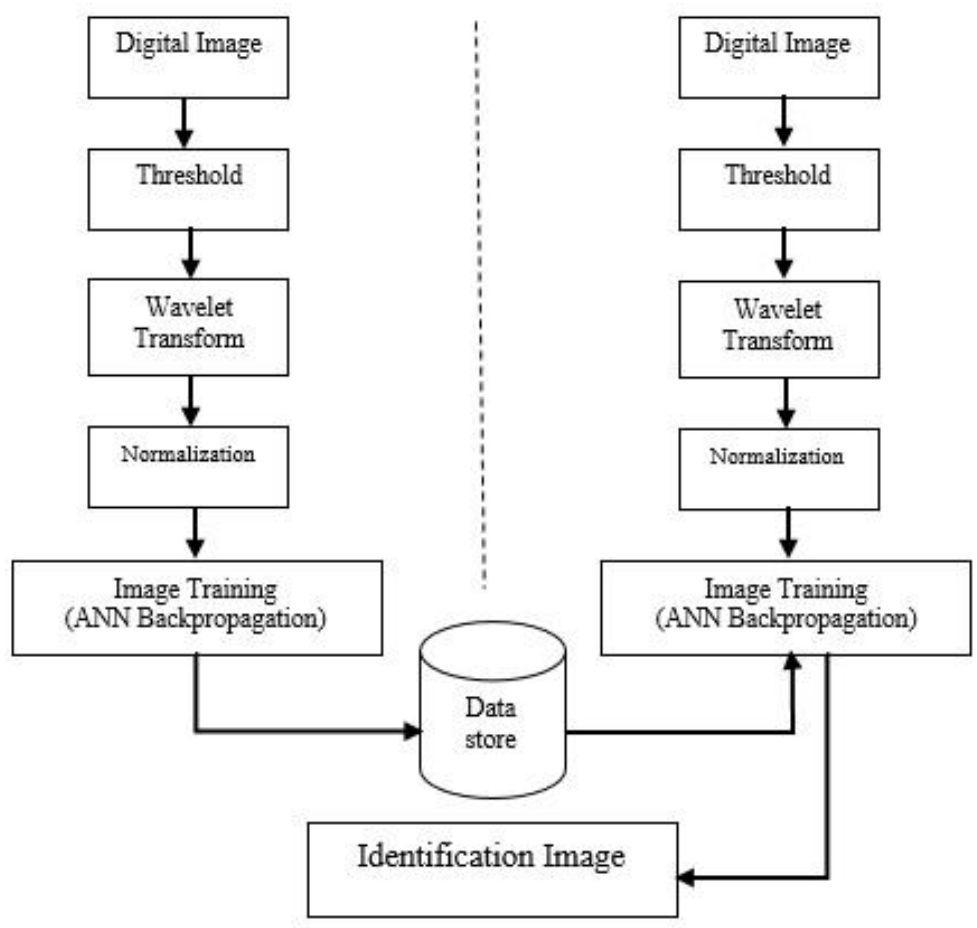

Figure 3. Handwriting Identification Flowchart

The handwritten image that will be sampled must first be converted into a digital image with the help of a scanner so that a digital image is obtained that is ready to be processed. Preprocessing is the initial step that will be given to the image, including the threshold and then it will be subjected to a wavelet transform to get important information in the image. After that the image will be normalized before entering the training phase. The image training phase is carried out on each ID / author in order to get the character and pattern of the handwritten image. The results of the signature character in the form of weights generated by Backpropagation ANN are then stored in the data store to then be compared with the test image. The test image can be taken from the same author with different raw images, so the test image will also go through the same preprocessing. The system is considered successful if the system can provide appropriate results. Figure 4 below represents the training phase on each ID/author. 


\section{International Journal of Applied Sciences and Smart Technologies}

Volume 3, Issue 2, pages 257-264

p-ISSN 2655-8564, e-ISSN 2685-9432

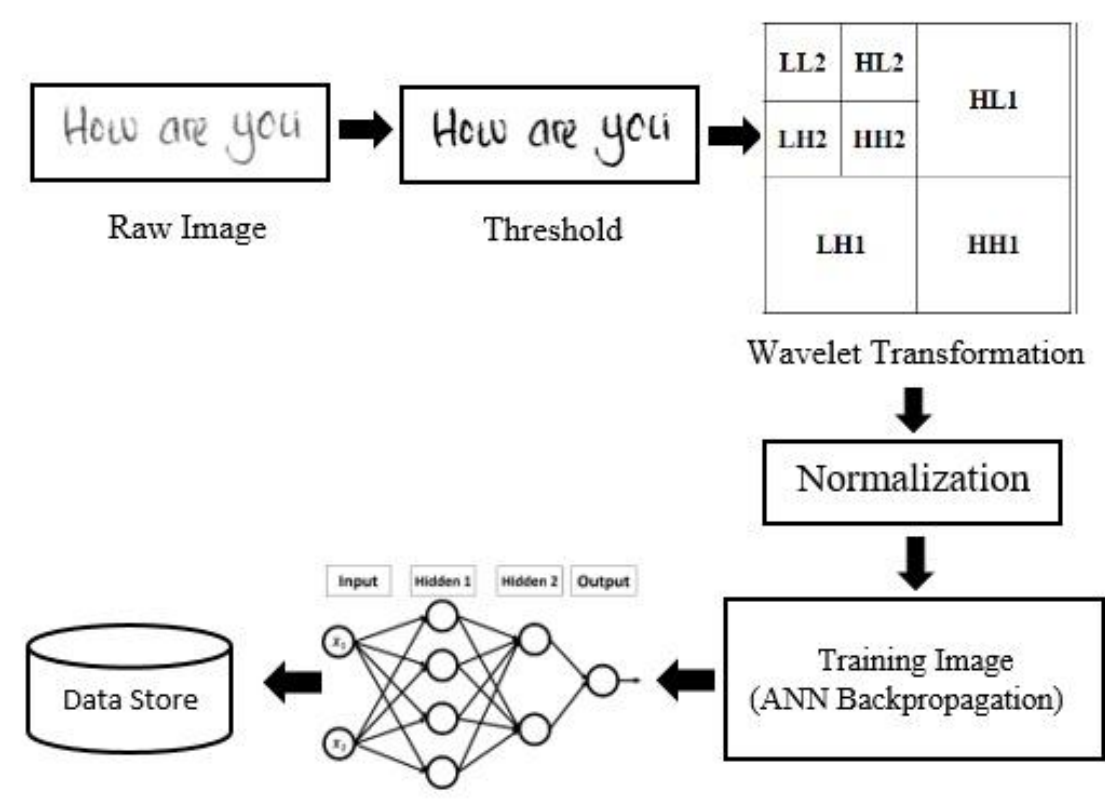

Figure 4. Training Phase

The system is considered reliable and successful if it can learn the character of each handwriting pattern so as to be able to distinguish the author's handwritten characters from one another. The indicator of the success of the system will be seen from the percentage of identification ability in all the IDs studied. Of course, here it is possible for errors to occur, so handwriting identification needs to be simulated using several parameters to get the optimal percentage. The consistency of a person's handwriting is also one of the influential factors so that the identification of this handwriting can also be added to several attributes to get more optimal results.

\section{Conclusion}

This study presents a method to identify handwriting using a Backpropagation Neural Network. ANN can be used in high complexity and is able to provide a progressive approach that is verified by various error rates. Utilization of the Backpropagation algorithm can minimize errors because there is a reverse/backward checking phase to correct errors. It is hoped that the design of this handwriting identification system can help minimize plagiarism among the public in all aspects. As 


\section{International Journal of Applied Sciences and Smart Technologies}

Volume 3, Issue 2, pages 257-264

p-ISSN 2655-8564, e-ISSN 2685-9432

for things that can be added from this research in the form of real-time identification that can provide direct results so that it is more effective and efficient.

\section{References}

[1] M. Sutajha, M. Sandeep, C. Aiswarya and B. Mounika, "Hand Writing Recognition System Based on Neural Network," International Journal on Innovative Technology and Exploring Engineering (IJITEE), 9 (1), 4977-4980, 2019.

[2] R. Ahuja and L. Duhan, "Optimized Multi Model Biometric Based Human Authentication Using Deep Neural Network," International Journal of Recent Technology and Engineering (IJRTE), 8 (3), 280-290, 2019.

[3] P. B. Nair, A. M. Johnson, A. M. Alex and A. Sebastian, "Android App for Handwriting Analysis Using Deep Learning," Journal of Communication Engineering and Its Innovations, 5 (3), 16-21, 2019.

[4] S. Aqab and M. U. Tariq, "Handwriting Recognition using Artificial Intelligence Neural Network and Image Processing," International Journal of Advanced Computer and Application (IJACSA), 11 (7), 137-146, 2020.

[5] P. G. Patil and R. S. Hegadi, "Offline Handwritten Signature Classification Using Wavelet and SUpport Vector Machines," International Journal of Engineering Science and Innovative Technology (IJEAT), 2 (3), 573-589, 2013.

[6] P. Divyasri, K. Depti and D. S. Rao, "Signature Analysis of Centrifugal Fan Response Due to Unbalance Using Wavelet Analysis," International Journal of Advance Research in Science and Engineering, 3 (7), 205-213, 2014.

[7] Suma'inna, "Detection of Cardiac Abnormalities Based on ECG Pattern Recognition Using Wavelet and Artificial Neural Network," Far East Journal of Mathematical Sciences, 76 (1), 111-122, 2013. 